www.jmscr.igmpublication.org

Impact Factor 5.84

Index Copernicus Value: 71.58

ISSN (e)-2347-176x ISSN (p) 2455-0450

crossref DOI: _https://dx.doi.org/10.18535/jmscr/v5i9.139

\title{
Tongue Crenation (Scalloped Tongue) - Case Report
}

\author{
Author \\ Khurshid A Mattoo ${ }^{1 *}$ \\ ${ }^{1}$ Assistant Professor, Department of Prosthetic Dental Sciences, College of Dentistry, \\ Jazan University, (KSA) \\ * Corresponding Author \\ Email:drkamattoo@rediffmail.com
}

\begin{abstract}
Multifunctional organ like tongue is an important indicator of underlying systemic diseases. However, sometimes under physiological influences, the tongue alters in its shape and size and appears clinically same as it appears under pathological state. An elderly male patient reported for replacement of missing natural teeth. Medical, dental, drug and psychosocial history were insignificant. The tongue had multiple indentations that conformed to the pattern of the surrounding teeth. A cast partial denture was indicated for both maxillary and mandibular arch. Factors leading to overgrowth of the tongue under physiological influences are discussed.

Keywords: lingua indentation, crenelation, amyloidosis, macroglossia, phonetics.
\end{abstract}

\section{Introduction}

The tongue is a highly mobile, basically muscular organ whose functions includes speech, mastication, swallowing, taste, expression, reflects the systemic status and helps with the dentogenesis and stability of the dental arches. The tongue is divided by a median septum (fibrous) into two halves and each half contains intrinsic and extrinsic muscle groups. The form or shape of the tongue within any organism is correlated to the function of the tongue. Abnormalities of tongue include primarily due to developmental or functional disturbances like macroglossia, microglossia, fissured tongue, ankyloglossia, aglossia and bifid tongue. Abnormalities secondary to systemic disorders are numerous and include conditions like scarlet fever, syphilis, tuberculosis, candidiasis, amylodoisis, blastomycosis, vitamin and mineral deficiencies, peripheral vascular diseases (diabetes, systemic lupus erythematosus), drugs (cyclosporine, antibiotics) ${ }^{1-6}$ and various premalignant lesions, conditions and benign and malignant tumors. ${ }^{7,8}$

Physiological condition as a result of form versus function takes the form of crenated tongue (crena notch latin). Also called lingua indentata, pie crust tongue, scalloped or crenulated tongue, the condition is a description of a tongue form whereby indentations occur laterally due to compression against the adjacent teeth. This article presents a case of a scalloped tongue which resulted due to absence of multiple teeth, wherein the tongue encroached into edentulous spaces thus creating the indentations, and has rarely been reported in the literature 


\section{Case Report}

An elderly male in his fifties reported to the department of Prosthodontics for replacement of missing natural teeth. The patient was a laborer by occupation and reported no medical problems. The patient had never been to any dentist while he used to get his teeth extracted by quacks. No evidence of developmental problems or any clinical feature that would indicate hormonal, infectious or neoplastic underlying problem. Extra oral examination revealed normal features of salivary gland, lymph nodes, facial features and temperomandibular joint. Intra orally the clinical features included generalized periodontitis, staining, plaque and calculus in relation to mandibular anterior teeth. Maxillary arch presented a Kennedy class 2 modification 1 while mandibular arch presented a Kennedy class 3 modification 2 partial edentulous space (Fig.1 a). A striking feature of intra oral examination was the patient's tongue which was irregularly crenulated following exactly the pattern of partial edentulous mandibular spaces (Fig. 1b). The tongue was enlarged on the right side while loosing the border definition on the same side. Due to enlargement of the tongue on the right side, it appeared scalloped with grooves and fissures which exactly corresponded to the impressions of the natural teeth (Fig1. c). The enlarged portion was more pronounced between mandibular premolar and molar on the right side. The mucosa over the tongue as well as lateral border presented a normal clinical picture with no evidence of oedematous, inflamematory, fibrous or neoplastic changes. Patients pronunciation was although affected, especially the linguo dental sounds while there was some difficulty in understanding speech that were linguo palatal in expression.

Some of the remaining teeth exhibited wear while maxillary posterior teeth exhibited a normal cuspal inclination. The patient was referred to department of oral medicine and diagnosis for screening of underlying systemic diseases which was not found to exist. Various treatment options advised to the patient included cast partial denture while mandibular dentition was advised for cast partial denture with surveyed crowns. The patient refused both treatments because of the time associated with such fabrication.

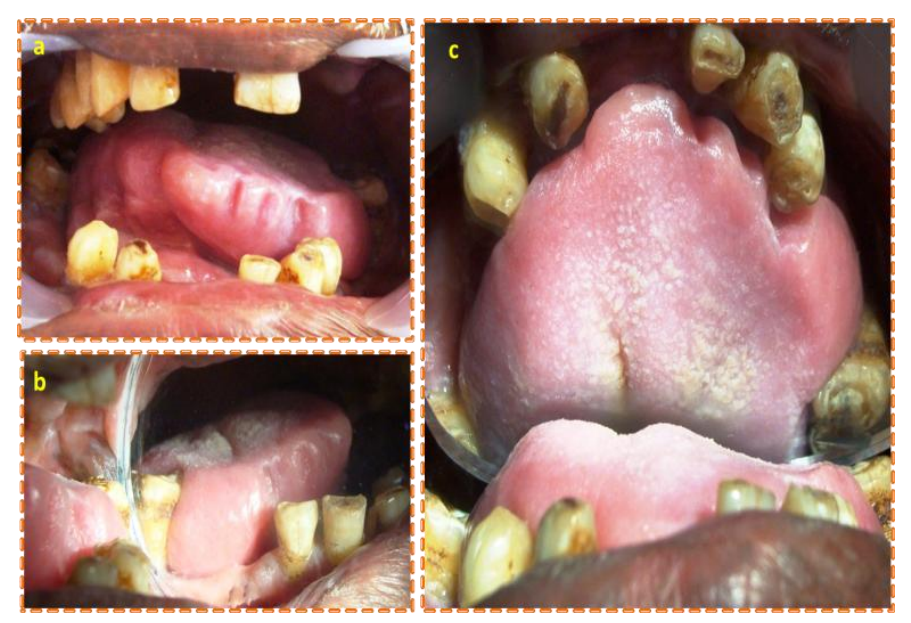

Figure 1: (a) Frontal view (b) Side view demonstrating largest portion of enlarged tongue (c) Superior view (note relationship of scalloping pattern with teeth

\section{Discussion}

Various terms of crenated tongue signify a common clinical, physiologic normal condition of the tongue which is not a disease of any form, but results from a combination of factors which are size of the tongue, status of the existing dentition and the pressure applied on the tongue by the surrounding teeth. Enlarged tongue, or macroglossia can be developed or can be due to systemic conditions like hypothyroidism, sarcoidosis, amyloidosis etc. in such cases, diagnosis is achieved by underlying history and varying clinical picture of the tongue. Obstructive sleep apnea being an exception in which tongue scalloping is present without evident macroglossia. ${ }^{9}$ Presence of teeth is an essential element of causing the crenation of the tongue. When a person is completely edentulous, then the tongue is less likely to possess fissures or crenulated appearance which is why, in such cases, enlargement of the tongue should be thoroughly associated for detection of underlying disease. In this case the patient had Kennedy class 3 partial edentulous situation in both arches. The crenulation of the tongue conformed exactly to the relation between the teeth and the edentulous spaces. The 
tongue was more enlarged on the right side between the first premolar and the last molar. Sequence of loss of teeth in that region was earlier than the rest of the teeth, which were approximately more than five years. Longstanding, non replacement of teeth, thus allows the tongue to grow in the edentulous space resulting in deepening of scalloping. This should not be seen as scalloping that is caused under normal circumstance which is associated with parafunctional habits like bruxism or any related anxiety disorder.

\section{Conclusion}

Crenation of the tongue can be either physiological in nature or due to underlying systemic disease. Careful history and clinical examination should be first done to differentiate between a physiological and pathological state. Once a normal variation is ruled out, only then further diagnostic testings should be focused.

\section{Acknowledgements}

The authors would like to acknowledge the cooperation provided by the staff of the department of oral medicine and diagnosis.

\section{References}

1. Tyldesley WR, Field A, Longman L (2003). Tyldesley's Oral medicine (5th ed.). Oxford: Oxford University Press. p. 195.

2. Ghom (2005). Textbook of Oral Medicine. Jaypee Brothers Publishers. p. 477

3. Byrd JA, Bruce AJ, Rogers RS. Glossitis and other tongue disorders. Dermatol Clin. 2003;21(1):123-34

4. Desport E, Bridoux F, Sirac C, Delbes S, Bender S, Fernandez B, et al. AL amyloidosis. Orphanet J Rare Dis. 2012;7:54.

5. Angiero F, Seramondi R, Magistro S, Crippa R, Benedicenti S, Rizzardi C, et al. Amyloid deposition in the tongue: Clinical and histopathological profile. Anticancer Res. 2010;30(7):3009-14.
6. Reamy BV, Derby R, Bunt CW. Common tongue conditions in primary care. Am Fam Physician. 2010;81(5):627-34.

7. Bhalavat RI, Mahantshetty UM, Tole S, Jamema SV. Treatment outcome with lowdose-rate interstitialbrachytherapy in earlystage oral tongue cancers. J Cancer Res Ther. 2009; 5(3):192-7.

8. Sessions DG, Spector GJ, Lenox J, Haughey B, Chao C, Marks J. Analysis of Treatment Results for Oral Tongue Cancer. The Laryngoscope 2002; 112:616-25.

9. Weiss TM, Atanasov S, Calhoun KH. The association of tongue scalloping with obstructive sleep apnea and related sleep pathology. Otolaryngol Head Neck Surg. 2005;133(6):966-71 